\title{
Warfarin-induced gastric intramural hematoma
}

\author{
Ricardo Humberto de Miranda Félix ${ }^{1}$, Breno Victor Tomaz Galvão ${ }^{2}$, Aécio Flávio Teixeira Góis ${ }^{1}$
}

${ }^{1}$ Department of Internal Medicine, Federal University of São Paulo, São Paulo, SP

2Department of Radiology, Federal University of São Paulo, São Paulo, SP

The authors and departments are attributed to the Federal University of São Paulo, SP

Study conducted at Universidade Federal de São Paulo, São Paulo, SP

Correspondence:

Universidade Federal de São Paulo

R. Napoleão de Barros, 715

São Paulo, SP

Cep: 04024-002

Conflict of interest: none

http://dx.doi.org/10.1590/1806-9282.60.01.005

\section{INTRODUCTION}

Gastric intramural hematoma is extremely rare. Most cases of gastrointestinal tract hematomas are located in the esophagus or in the duodenum, being usually associated with anticoagulant therapy, coagulopathy, trauma, ulcer, amyloid angiopathy, repeated vomiting, complications of endoscopy and idiopathic disease. ${ }^{1-7} \mathrm{~A}$ case of gastric hematoma in a patient using warfarin in a conservative measure will be described in this short report.

A 41-year-old female patient came to a clinic complaining of four-day nausea and abdominal pain. She referred to a mild pain, which was intermittent, burning, radiating to the left hypochondrium, associated with nausea and one vomiting event. There was no history of alcohol use, tobacco smoking or overeating. The past medical history had shown leiomyosarcoma in the right kidney one year prior to admission, treated with right nephrectomy. She had also had thrombosis of inferior vena cava extending to both common iliac veins to the right atrium beyond pancreatic and adrenal metastasis. She had undergone maintenance treatment with warfarin and palliative chemotherapy with docetaxel and gemcitabine.

Initially she had been medicated with omeprazole and tramadol with a good relief of symptoms. Laboratory data have shown hemoglobin $10.1 \mathrm{~g} / \mathrm{dL}(12-15.5)$, leucocytes $28.4 \times 10^{9} / 1(3.5-10.5)$, thrombocytes $148 \times 10^{9} / 1$ (150-450), amylase $52 \mathrm{U} / \mathrm{l}(0-99)$, creatinine $1.41 \mathrm{mg} / \mathrm{dL}$ (0.5-0.9), INR 3.51 (0.8-1.2) and normal values of markers of myocardial necrosis and hepatic injury. After a first two-day assessment, she developed severe epigastric pain, pale skin, sudoresis and lipothymia. Physical examination had shown a distressed woman with blood pressure of $74 / 38 \mathrm{mmHg}$, a pulse rate of 110 per minute, a respiration rate of 26 per minute and normal temperature. Further physical examination presented pain in epigas- tric palpation. Electrocardiogram showed sinusal tachycardia with hemoglobin levels of $3.9 \mathrm{~g} / \mathrm{dL}$, creatinine 2.52 $\mathrm{mg} / \mathrm{dL}$ and INR 8.76. She had been resuscitated by saline infusion and red blood cells transfusion. A computed tomography scan of the abdomen demonstrated a $17.6 \times$ $12.4 \times 7.08 \mathrm{~cm}$ large fluid collection in the stomach, heterogenic with hyper attenuating areas in between, consistent with hematoma, which after injection of contrast medium, had shown foci of active bleeding (Figure 1).

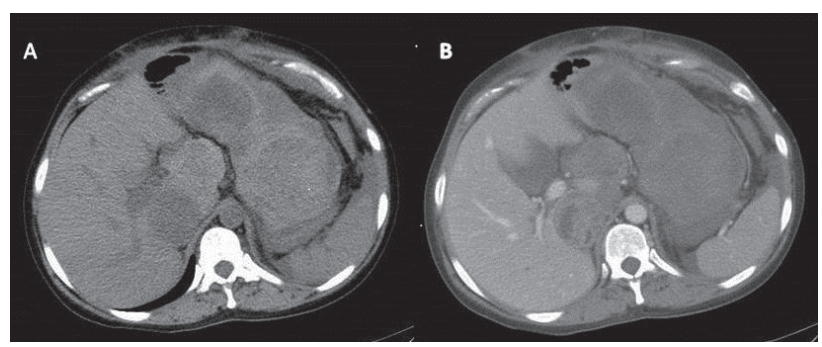

\section{FIGURE 1}

The oral anticoagulation was discontinued and fresh frozen plasma transfusion was performed. The patient responded to these initial procedures well, being hemodynamically stable with corrected anemia and coagulation tests. She was treated conservatively with fasting and intravenous proton pump infusion for 3 days. She was discharged with oral feeding five days after hospitalization.

Gastric intramural hematoma after anticoagulant therapy is an uncommon and extremely rare disorder with few reports published in the literature. ${ }^{1-6}$ Hematoma can develop in the submucosal layer and the proper muscle layer of the gastrointestinal wall. ${ }^{8}$ It can be accompanied by hematemesis, nausea, vomiting, epigastric pain that can simulate acute coronary syndrome. ${ }^{4}$ 
In this present case study, gemcitabine had increased the effects of warfarin, contributing to the formation of hematoma.

CT scanning has become the test of choice in the diagnostic procedure. Gastrointestinal hematoma may be described as well-circumscribed, high-density homogeneous masses, lacking signs of calcification and infiltration in other organs. ${ }^{9,10}$ Angiography can be performed, according to therapeutic goals, especially when there is evidence of active bleeding. ${ }^{2}$

There is no standard therapy and most cases are managed conservatively with blood transfusion and anticoagulation reversal. ${ }^{1,3-6}$ In hematoma with substantial bleeding and a trend toward enlargement, transcatheter arterial embolization or surgical therapy (including total gastrectomy) may be indicated. ${ }^{2,7}$ Surgery is also a treatment of choice, especially in patients with hematomas which are difficult to distinguish from tumors. ${ }^{2}$

\section{References}

1. Dhawan V, Mohammed A, Fedorak RN. Gastric intramural hematoma: a case report and literature review. Can J Gastroenterol. 2009; 23:19-22.

2. Imaizumi H, Mitsuhashi T, Hirata M, Aizaki T, Nishimaki H, Soma K, et al A giant intramural gastric hematoma successfully treated by transcatheter arterial embolization. Intern Med. 2000; 39:231-4.

3. Hurtado-Doce AI, Solla-Buceta MA, Galeiras-Vázquez RM. Spontaneous intramural gastric hematoma in an anticoagulated patient. Rev Esp Enferm Dig. 2011; 103:496.

4. Leborgne L, Mathiron A, Jarry G. Spontaneous intramural gastric haematoma as a complication of oral anticoagulant therapy mimicking acute myocardial infarction. Eur Heart J. 2007; 28:1804.

5. Durward QJ, Cohen MM, Naiman SC. Intramural hematoma of the gastric cardia. Am J Gastroenterol. 1979; 71:301-5

6. Balthazar EJ, Einhorn R. Intramural gastrointestinal hemorrhage. Clinical and radiographic manifestations. Gastrointest Radiol. 1976; 3:229-39.

7. Hui J, AhChong AK, Mak KL, Chiu KM, Yip AW. Spontaneous intramural hematoma of stomach. Dig Surg. 2000; 17(5):524-7.

8. Bailey WC, Akers DR. Traumatic intramural hematoma of the duodenum in children. A report of five cases. Am J Surg. 1965; 110:695-703.

9. Plojoux $\mathrm{O}$, Hauser H, Wettstein P. Computed tomography of intramural hematoma of the small intestine: a report of 3 cases. Radiology. 1982; 144:24.

10. Scheward SE, Davis M, Amparo EG, Gogel HK. Intramural hemorrhages simulating gastric neoplasm. Gastrointest Radiol. 1988; 13:102-4. 